\title{
Elastic stiffness coefficients of thenardite and their pressure and temperature dependence
}

D. Arbeck: Institut für Geowissenschaften, Abt. Kristallographie

Goethe Universität Frankfurt, Altenhöferallee, 1, 60438 Frankfurt am Main, Germany

E. Haussühl: Institut für Geowissenschaften, Abt. Kristallographie

Goethe Universität Frankfurt, Altenhöferallee, 1, 60438 Frankfurt am Main, Germany

V. Vinograd: Institut für Geowissenschaften, Abt. Kristallographie

Goethe Universität Frankfurt, Altenhöferallee, 1, 60438 Frankfurt am Main, Germany

B. Winkler: Institut für Geowissenschaften, Abt. Kristallographie

Goethe Universität Frankfurt, Altenhöferallee, 1, 60438 Frankfurt am Main, Germany

N. Paulsen: Institut für Geowissenschaften, Abt. Kristallographie

Goethe Universität Frankfurt, Altenhöferallee, 1, 60438 Frankfurt am Main, Germany

S. Haussühl: Institut für Kristallographie

Universität zu Köln, Zülpicher Str., 49b, 50674 Köln, Germany

V. Milman: Accelrys Inc., 334 Cambridge Science Park, Cambridge 6CB4 0WN, United Kingdom

J.D. Gale: Nanochemistry Research Institute, Department of Chemistry, Curtin University, GPO Box U1987, Perth, WA 6845, Australia

\begin{tabular}{l|l|r}
\hline \hline MS-ID: & arbeck@kristall.uni-frankfurt.de & 28. Oktober 2011 \\
Heft: / () &
\end{tabular}




\begin{abstract}
The elastic stiffness coefficients, $c_{i j}$, of orthorhombic $\mathrm{Na}_{2} \mathrm{SO}_{4}$ thenardite (space group Fddd) were measured with an ultrasonic plane wave technique at ambient temperature as a function of hydrostatic pressure in the range of $0.1-70 \mathrm{MPa}$. The variation of the $c_{i j}$ in the range of $1-5000 \mathrm{MPa}$ was studied with density functional theory (DFT) based calculations. The experimental results and the DFT calculations were used to derive a force-field model, which was then employed to compute lattice parameters and elastic stiffness tensors of thenardite and of two other $\mathrm{Na}_{2} \mathrm{SO}_{4}$ polymorphs as functions of the temperature based on quasi-harmonic lattice dynamics. The structural parameters of the three polymorphs measured at high temperatures are reproduced to within $1.7 \%$ by the present calculations. Phases II (space group $\mathrm{Pbnm})$ and III ( $\mathrm{Cmcm})$ appear to have significantly higher entropies than thenardite in agreement with their metastable formation at higher temperatures.
\end{abstract}




\section{Introduction}

Sodium sulphate, $\mathrm{Na}_{2} \mathrm{SO}_{4}$, exists in four different modifications that have been structurally characterised often labelled as I, II, III and V[? ]. Thenardite (phase V), which is stable at ambient conditions, is a major commodity chemical [?]. Its principal use is in processing wood pulp for paper production. The knowledge of the phase equilibria of thenardite, particularly on its transformation into mirabilite $\left(\mathrm{Na}_{2} \mathrm{SO}_{4} \cdot 10 \mathrm{H}_{2} \mathrm{O}\right)$, are important for understanding mechanisms of weathering and damage of buildings and monuments of cultural heritage [? ? ] Phase relations between the four polymorphs have been studied by ? ? ]. Vickers hardness, abrasive properties, thermal expansion, and elastic and thermoelastic coefficients at ambient pressure and temperature have been reported by ? ]. The present study is concerned with the elastic behaviour of thenardite and the other polymorphs at higher pressures and temperatures. Here we report the results of measurements of the piezoelastic coefficients of thenardite at moderately high pressures using a technique based on measuring the speed of an ultrasonic wave traveling across plane parallel plates. These studies are complemented with resonant ultrasound spectroscopic experiments and with DFT-based and empirical force-field atomistic calculations.

The development of a force-field model for a material like thenardite is not straightforward. The conventional static fitting procedure, in which the force-field parameters are adjusted in order to reproduce structural data and elastic stiffness coefficients, may not be appropriate given that thenardite is a soft material. Hence, thermal expansion and zero point motion should not be ignored and the structural data collected at ambient temperature cannot be used as constraints in the static lattice energy minimisation procedure. Therefore, the forcefield fitting performed here was based on a different strategy that explicit accounts for lattice dynamical effects. We show that the use of a free energy minimisation derived set of input parameters allows the structures of the three phases to be modelled in a correct manner.

The paper is organised as follows. First, we introduce the theory behind the ultrasonic measurements performed here. Then we describe further details of the experimental setup. Next, we determine the same properties by means of atomistic calculations and compare the experimental results with the theoretically obtained values.

\section{Crystal structure}

First analyses of the crystal structure of $\mathrm{Na}_{2} \mathrm{SO}_{4}(\mathrm{~V})$ were performed by ? ] and ? ]. The structural model proposed by ? ] was later confirmed by crystal structure refinements of natural thenardite by ? ] and the synthetic anhydrous sodium sulphate by ? ]. The crystal structure can be described in terms of distorted $\mathrm{NaO}_{6}$ octahedra and nearly regular $\mathrm{SO}_{4}$ tetrahedra (Figure ??).

\section{Ultrasonic plane wave techni- que}

The physical properties reported here are given with respect to either the crystallographic or Cartesian reference systems, where the latter is denoted by a prime. The elastic stiffness tensor can be determined from basic elasto-dynamic equations which relate the propagation velocity of an ultrasonic wave, $v=f \cdot \lambda$, to its displacement vector, $\xi_{k}[?]$;

$$
\left(-\rho v^{2} \delta_{i k}+c_{i j k l} g_{j} g_{l}\right) \xi_{k}=0,
$$

where $g_{j}, g_{l}$ are the components of the propagation direction and $\delta_{i k}$ is the Kronecker symbol. The equation can be solved for the eigenfrequencies for any $\xi_{k}$-vector. Then, knowing the displacement vector, one obtains the relationship between the velocity and the $c_{i j}$ depending on the components of the displacement vector. For example, for a longitudinal ultrasonic wave, which travels perpendicular to the face of a $(110)^{\prime}$ plate of a crystal with orthorhombic symmetry, one obtains:

$$
\begin{aligned}
\rho v^{2}= & \frac{1}{4}\left(c_{11}+c_{22}+c_{66}\right) \\
& +\frac{1}{2} \sqrt{\frac{1}{4}\left(c_{11}-c_{22}\right)^{2}+\left(c_{12}+c_{66}\right)^{2}}
\end{aligned}
$$

Similarly, for a transverse wave travelling perpendicular to the $(110)^{\prime}$ face with the displacement vectors [110] and [001] one obtains; 


$$
\begin{aligned}
\rho v^{2}= & \frac{1}{4}\left(c_{11}+c_{22}+c_{66}\right) \\
& -\frac{1}{2} \sqrt{\frac{1}{4}\left(c_{11}-c_{22}\right)^{2}+\left(c_{12}+c_{66}\right)^{2}}
\end{aligned}
$$

and

$$
\rho v^{2}=\frac{1}{2} c_{44}+\frac{1}{2} c_{55}
$$

respectively.

The resonance frequencies can be measured through the excitation of mechanical oscillations in the sample, which typically is a parallel plate, using an ultrasonic transducer. The ultrasonic wave generated at the face of the plate, which is in contact with the transducer, travels through the crystal and is reflected by the upper face of the plate. It returns a part of its mechanical energy to the transducer via the piezoelectric effect. Resonance frequencies can be detected by measuring the phase angle between the current and the voltage [? ] (Figure ??). The difference in the frequencies between two neighbouring resonance peaks, $\Delta f$, and the thickness of the sample, $d$, are related to the velocity of the ultrasonic wave [?]:

$$
v=2 \cdot d \cdot \Delta f
$$

For an orthorhombic crystal $c_{11}, c_{22}$ and $c_{33}$ can be directly calculated from the velocities of three longitudinal waves excited in the directions perpendicular to the (100), (010) and (001) planes:

$$
\begin{aligned}
& c_{11}=\rho \cdot\left(2 \cdot d_{100} \cdot \Delta f_{100}\right)^{2} \\
& c_{22}=\rho \cdot\left(2 \cdot d_{010} \cdot \Delta f_{010}\right)^{2} \\
& c_{33}=\rho \cdot\left(2 \cdot d_{001} \cdot \Delta f_{001}\right)^{2}
\end{aligned}
$$

In the case of an externally applied hydrostatic pressure, these equations must be modified by taking into account the change in the density and in the thickness of the plate [?]:

$$
\begin{aligned}
c_{i j}= & \rho_{0} \cdot\left(1+\sum_{i j} s_{i i j j} \cdot \Delta p\right) \\
& \cdot\left(2 \cdot d_{0} \cdot\left(1-\sum_{k} s_{i j k k} \cdot \Delta p\right) \cdot \Delta f\right)^{2},
\end{aligned}
$$

where $\rho_{0}$ is the density and $d_{0}$ is the thickness of the sample at ambient pressure, respectively. The $s_{i j k l}$ are the elements of the inverse matrix of the elastic stiffness coefficients, $c_{i j k l}$, at ambient pressure.

The determination of the piezoelastic coefficients

$$
p_{i j}=\frac{\mathrm{d} c_{i j}}{\mathrm{~d} p}
$$

requires that the $c_{i j}$ are measured at several pressures. Equation 10 is often written in the form [? ]:

$$
p_{i j}=p_{i j}^{*} \cdot c_{i j}
$$

where

$$
\begin{aligned}
p_{i j}^{*} & =\frac{\frac{\mathrm{d} \log c_{i j}}{\mathrm{~d} p}}{\underbrace{\sum_{i j} s_{i i j j}}_{\text {bulk compressibility }}-\underbrace{2 u_{1 i} u_{1 j} \sum_{k} s_{i j k k}}_{\text {linear compressibility }}}+2 \frac{\overline{\Delta f}}{\bar{f} \cdot \Delta p},
\end{aligned}
$$

with which the measurement of the $p_{i j}$ can be reduced to the measurement of the shift, $\overline{\Delta f}$, in the averaged frequency, $\bar{f}$, due to the applied hydrostatic pressure, $\Delta p$.

\section{Resonant ultrasound spectros- copy}

Resonant ultrasound spectroscopy (RUS) is based on the determination of eigenfrequencies of freely vibrating crystals [? ]. The spectra, typically obtained in the 100 $1000 \mathrm{kHz}$ frequency range cannot be directly transformed to yield the $c_{i j}$. Instead, the components of the elastic stiffness tensor have to be obtained from the measured resonance frequencies via a non-linear least-squares refinement procedure in which the observed values are compared to theoretical ones. The theoretical resonance 
frequencies are obtained by solving a general eigenvalue problem, whose rank is equal to the number of basis functions needed for the description of the components of the displacement vector [?]:

$$
\boldsymbol{\Gamma} a=\left(\rho \omega^{2}\right) \mathbf{E} a
$$

The eigenvalues are $\lambda=\rho \omega^{2}$ and the eigenvectors, $a$, are the expansion coefficients of the matrices $\mathbf{E}$ of the potential energy and $\boldsymbol{\Gamma}$ of the corresponding kinetic energy. In the least-squares refinement procedure the quantity

$$
\chi=\sum_{i=1}^{n} w_{i} \cdot\left(\omega_{i \text { cal }}^{2}-\omega_{i \text { obs }}^{2}\right)^{2}
$$

is calculated for $n$ eigenmodes with the resonance frequencies $f_{i}=\omega_{i} / 2 \pi$ and minimized by varying the elastic stiffness coefficients. The $w_{i}$ are the weights calculated by assuming an experimental error of $\pm 0.05 \mathrm{kHz}$ for each of the observed resonance frequencies [? ]. In order to minimize errors due to truncation effects, up to several thousand normalized Legendre polynomials are used in the development of the displacement vector. A more detailed description of the RUS technique can be found elsewhere (e.g. ?] or ? ]).

\section{$5 \quad$ Experimental}

\subsection{Sample preparation}

Optical quality single crystals of thenardite with dimensions of $50 \times 30 \times 25 \mathrm{~mm}^{3}$ were grown from an aqueous solution. The measurements of the piezoelastic coefficients were performed with plane parallel plates with approximate dimensions of $14 \times 12 \times 3 \mathrm{~mm}^{3}$ (Figure ??). The parallelism of the plates is better than $3 \mu \mathrm{m}\left( \pm 0.02^{\circ}\right)$. The resonant ultrasound spectroscopy measurements were performed with a rectangular sample of dimensions $7.658(5) \times 5.746(1) \times 9.022(3) \mathrm{mm}^{3}$. The samples were cut using a low speed wire saw and polished using a slurry of corundum powder $(10 \mu \mathrm{m})$ and propanol. The faces of the samples used for the resonant ultrasound studies were oriented parallel to the faces of the orthorhombic cell. The deviation from the ideal crystallographic orientation was controlled by Xray diffraction, using a Bragg-Brentano configuration, and was smaller than $0.5^{\circ}$. The physical properties were described in a Cartesian reference system with the basic vectors $\overrightarrow{e_{i}}$ parallel to the crystallographic basic vectors $\overrightarrow{a_{i}}$. Their magnitudes are $\left|\overrightarrow{a_{1}}\right|=5.868(1) \AA$, $\left|\overrightarrow{a_{2}}\right|=12.302(2) \AA$ and $\left|\overrightarrow{a_{3}}\right|=9.829(1) \AA$. This setting is the same as that used by ? ]. The plates used for the measurement of the piezoelastic coefficients were oriented parallel to the crystallographic faces (100), (010), (001), (110)', (101)', (011)' and $(111)^{\prime}$.

\subsection{Density measurement}

The density of $\mathrm{Na}_{2} \mathrm{SO}_{4}$ at $295 \mathrm{~K}$ was measured using the buoyancy method in paraffin oil. A Sartorius ME235S analytical balance was used. A value of $2.666(5) \mathrm{g} / \mathrm{cm}^{3}$ was obtained by averaging over several measurements.

The theoretical X-ray density is $2.659(2) \mathrm{g} / \mathrm{cm}^{3}$. The $\mathrm{X}$-ray density is often observed to be $0.5-1 \%$ higher than values obtained from buoyancy measurements due to defects. In our case, the two values agree within errors, indicating the excellent quality of our samples.

\subsection{Measurement of the piezoelastic constants with the plane parallel plate method}

The sample was mounted on ultrasound transducer using paraffin oil or resin, which in turn was connected to an analyzer (Hewlett Packard HP 4194A network analyzer) as shown in Figure ??. The analyzer was used to generate an oscillating electrical signal (which leads to mechanical oscillation of the transducer) and to record the response signal from the coupled system of the transducer and the sample. The speed of ultrasound waves inside the crystal was calculated by measuring the frequency difference between the maxima of the impedance of the transducer as was described by ? ].

The frequency was varied from 1 to $18 \mathrm{MHz}$ in steps of $1 \mathrm{kHz}$. Measurements of the elastic stiffness constants were performed at $298 \mathrm{~K}$ in the pressure range of $0.1-70$ $\mathrm{MPa}$ using an autoclave equipped with an external cooling and an internal heating device. Pressure and temperature were controlled with two process controllers 
(Eurotherm-2604) to within $1 \mathrm{~K}$ and $1 \mathrm{MPa}$ and monitored automatically. The heating element inside the autoclave was a corundum tube onto which a heating coil was wrapped. The sample was mounted in the centre of the tube. The temperature was controlled with a thermocouple of type ' $\mathrm{K}$ ' fixed at the distance of about $1 \mathrm{~cm}$ from the sample (Figure ??).

A single measurement at constant pressure required about two hours. The pressure was increased from $0.1 \mathrm{MPa}$ to $70 \mathrm{MPa}$ in steps of $10 \mathrm{MPa}$. Figure ?? shows the acoustic resonance spectrum collected at $298 \mathrm{~K}$ and $0.1 \mathrm{MPa}$. With the pressure increase the resonances shift to lower frequencies and their amplitudes decrease. The piezoelastic constants were obtained from the averaged frequency shifts due to the pressure changes. The measured piezoelastic cooefficients are given in Table ??.

\subsection{Resonant ultrasound spectroscopy, RUS}

The elastic properties of thenardite were also studied with a custom made RUS-device. The samples were gently fixed between two ultrasound transducers, where the lower transducer acts as the ultrasound generator and the upper one as the ultrasound detector (Figure ??). The force acting on the opposed corners of the samples was less than $0.05 \mathrm{~N}$. This ensured that the experimental setup closely fulfilled the conditions of a freely vibrating body. The resonance spectra were collected at $295 \mathrm{~K}$ in the frequency interval of $50-1000 \mathrm{kHz}$ with a resolution of $0.01 \mathrm{kHz}$ using a network analyzer (HP 4394A from Agilent). The sample was mounted in four different orientations and four RUS spectra were collected. Figure ?? shows an example of an experimentally obtained resonance spectrum. The elastic constants were retrieved from the measured resonance frequencies by the non-linear least-squares procedure described above.

The experimental data obtained here with the ultrasonic techniques are in good agreement with the experimental data from ? ], measured with an improved Schaefer-Bergmann technique [? ]. The deviation between the elastic stiffness coefficients measured with the different techniques is below $5 \%$, except for the $c_{12}$, where the literature and the RUS values are $12 \%$ larger than the present plane wave parallel plate (PPP) measurement, and for the $c_{23}$, where the RUS value is
$10 \%$ larger than the PPP value. The measured $c_{i j}$ are compared to the other data sources in Table ??.

\section{Quantum mechanical calculati- ons}

The present quantum mechanical calculations were designed to provide an independent test for the experimentally determined values of the piezoelastic coefficients and to provide estimates of the structural parameters of phases II, III and V. At the same time, we wanted to investigate the accuracy with which piezoelastic coefficients for a compressible compound can be obtained with an atomistic model based on density functional theory, DFT. The calculations were performed with academic and commercial versions of the CASTEP program [? ? ? ] using a generalized gradient approximation, GGA based on the PBE exchange-correlation functional [?]. For all calculations ultrasoft pseudopotentials were used, with a small core for sodium, and the maximum cut-off energy of the plane waves was $410 \mathrm{eV}$. In addition to the cut-off energy, the quality of the calculations is determined by the density of the grid of $k$-points in the Brillouin zone. Here distances between the points were less than $0.026 \AA^{-1}$. The wave vectors for the sampling points were chosen according to the scheme proposed by ? ]. Full geometry optimisation calculations were performed. Structures were considered to be converged when the largest residual component of the stress tensor was less than $0.02 \mathrm{GPa}$ and the largest residual force acting on an atom was less than $0.01 \mathrm{eV} / \AA$. The elastic stiffness coefficients were obtained as the proportionality constants between the applied strains and the resultant stresses. Strain patterns were imposed with several magnitudes up to a distortion of $0.2 \%$ of the lattice parameters, and after geometry optimisation of the internal parameters the resultant stresses were computed. The present calculations are restricted to the athermal limit, in which temperature effects and zero-point motions were neglected. The geometry optimisation of the phases II, III and V has also been performed with the PBEsol functional (? ]), which offers an improved description of equilibrium properties of densely packed solids. Figure ?? compares the predicted interatomic distances 
with those of the experimental structure [? ]. Typically, the GGA-PBE based calculations predict structures which are slightly softer than experimental ones [? ]. However, the present results appear to be in a nearly perfect agreement with the structure determined by ? ]. Since thenardite is a soft material, this agreement implies that the thermal expansion of the experimental sample is nearly compensated by overestimation of the structural parameters due to the systematic underbinding typically exhibited by the PBE functional. Thus, we believe that the GGA-PBE calculations provide a realistic description of the elastic properties of the experimental sample at $298 \mathrm{~K}$. The $c_{i j}$ obtained from the quantum mechanical calculations are given in Table ??. The calculated and measured $c_{i j}$ and $p_{i j}$ are compared in Figures ?? and ??. Figure ?? further illustrates the relationship between the $c_{i j}$ and $p_{i j}$.

\section{$7 \quad$ Force-field calculations}

In addition to quantum mechanical methods we have explored the use of force-field calculations for the present system. While density functional techniques are only practical in the athermal limit, the use of a force-field approach makes it feasible to include the explicit effect of temperature and zero point energy on the structure and properties. There have been extensive force-field studies of sulphate minerals, though to date the majority have focused on alkaline earth sulphates, especially barite $\left(\mathrm{BaSO}_{4}\right)$ [? ] because of the interest in scale formation. The most general force-field model for this class of material is that of Allan et al [?] who fitted the structures and elastic constants of both alkali metal and alkaline earth sulphates. They employed a molecular mechanics description of the sulphate anion with an ionic model for the cations. Although a more recent model [?] has explored the use of polarisable oxygen ions, through the addition of a shell model, for a limited number of sulphates, this extra complexity is found not to improve the description of sulphate for the present system. Hence we have taken the model of Allan and co-workers as the starting point for force-field derivation here.

The objective of the current study is to be able to compute the structure and elastic properties of $\mathrm{Na}_{2} \mathrm{SO}_{4}$ polymorphs as a function of temperature. This can be achieved through the use of lattice dynamics via free energy minimisation within the quasi-harmonic approximation. Here we work within the Zero Static Internal Stress Approximation (ZSISA) [? ] in which the lattice parameters are minimised with respect to the free energy, while the internal degrees of freedom are optimised based on the potential energy such that the phonon frequencies are correctly computed for structures of zero force. All force-field calculations are performed using analytical first derivatives of the free energy [? ] as implemented within the program GULP [? ?]. The phonon densities of states are integrated across the Brillouin zone by using a symmetry reduced Monkhorst-Pack mesh [?] where the grid fineness is increased to achieve convergence of the free energy to a precision of $1 \mathrm{meV}$.

While it would be possible to directly employ the force-field parameters of Allan and co-workers in the present study, these were derived for use in athermal calculations (i.e. the effect of temperature is subsumed into the force-field parameters for ambient conditions). If this model were to be applied to free energy minimisation then there would be a double counting of the effect of temperature. For this reason we have chosen to refit the Na-O interaction in a manner that is specifically designed for use with explicit inclusion of thermal effects. Two approaches to this fitting procedure are possible. Firstly, it would be possible to use the athermal structural and elastic information obtained from density functional calculations as the observables to which the parameters could be fitted since vibrational contributions are explicitly absent. Because the elastic properties determined using the PBE exchange-correlation functional are already systematically underestimated with respect to the experimental values, and the inclusion of thermal effects will only further soften the elastic constant tensor, this strategy is not found to lead to a good model. Secondly, the experimental structural data and elastic constants can be directly fitted based on the relaxed fitting method [? ]. Here the parameters are varied to minimise the discrepancy between the free energy optimised structures and properties at the temperature corresponding to the experimental measurement. This second approach is the method that we have adopted. Here the structures of thenardite (phase V) and one of its polymorphs, phase III, have been fitted at temperatures of 293 and $463 \mathrm{~K}$, respectively, along with the 
elastic constants of thenardite as determined according to the experimental plane wave technique in the present work. This leads to the revised Na-O Buckingham parameters given in Table ??

\section{Results and Discussion}

Our experimental data show that the $c_{i j}$ s increase monotonously with pressure. This indicates that thenardite does not undergo a structural transition in the pressure range of 0.1 to $70 \mathrm{MPa}$.

Based on the DFT calculations Figure ?? shows the relative change in the calculated interatomic distances between 0 and $1000 \mathrm{MPa}$. This plot shows the near pressure-independence of the sulphur - oxygen and oxygen - oxygen distances. This suggests that the $\mathrm{SO}_{4}$ tetrahedra behave as nearly rigid units, whereas the compressional mechanism is mainly determined by the deformation of the $\mathrm{NaO}_{6}$ octahedra.

The evolution of the experimentally determined $c_{i j}$ with pressure is shown in Figure ??. This Figure again demonstrates the high degree of consistency between the experimentally determined and calculated data. The correlation between the experimentally and theoretically obtained values can be further illustrated using a graphic representation of longitudinal effects employing a tensor surface defined with the equation

$$
\begin{aligned}
F= & x_{i} x_{j} x_{k} x_{l} c_{i j k l} \\
= & u_{1 i} u_{1 j} u_{1 k} u_{1 l} c_{i j k l}\left|\vec{x}^{4}\right| \\
= & x_{1}^{4} c_{1111}+x_{2}^{4} c_{2222}+x_{3}^{4} c_{3333}+ \\
& x_{1}^{2} x_{2}^{2}\left(2 c_{1122}+4 c_{1212}\right)+x_{1}^{2} x_{3}^{2}\left(2 c_{1133}+4 c_{1313}\right)+ \\
& x_{2}^{2} x_{3}^{2}\left(2 c_{2233}+4 c_{2323}\right)+x_{1}^{3} x_{2} 4 c_{1112}+x_{1}^{3} x_{3} 4 c_{1113}+ \\
& x_{2}^{3} x_{1} 4 c_{2221}+x_{2}^{3} x_{3} 4 c_{2223}+x_{3}^{3} x_{1} 4 c_{3331}+x_{3}^{3} x_{2} 4 c_{3332}+ \\
& x_{1}^{2} x_{2} x_{3} 4\left(c_{1123}+2 c_{1213}\right)+x_{2}^{2} x_{1} x_{3} 4\left(c_{2213}+2 c_{2123}\right)+ \\
& x_{3}^{2} x_{1} x_{2} 4\left(c_{3312}+2 c_{3132}\right)
\end{aligned}
$$

where $x_{i}=u_{1 i}|\vec{x}|$ are the components of the radius vector $\vec{x}$, which points from the origin to the tensor surface. The $u_{1 i}$ are the cosines of the angle between $\vec{x}$ and the Cartesian vector $\overrightarrow{e_{1}}$. The representation surfaces of the longitudinal effects of the measured elastic and piezoelastic tensors are shown in Figures ?? and ??. The good correspondence between the surfaces constructed based on the calculated and experimental data shows that present DFT calculations are able to reproduce sufficiently accurately the major features of the compressional behaviour of thenardite.

Further details of the compressional mechanism and structure-property relations follow from the analysis of the shapes of the representation surfaces. The direction of the maximum of the longitudinal elastic stiffness, $c_{1111}^{\prime}(\mathbf{u})=u_{1 i} u_{1 j} u_{1 k} u_{1 l} c_{i j k l}$, where $u_{1 i}$ are the direction cosines, coincides with the direction [010] of the crystal structure (Table ??). The ratio $c_{1111}^{\prime}(\max ) / c_{1111}^{\prime}(\min )$ of $\approx 1.7$ indicates significant elastic anisotropy.

It is well known from empirical observations that the deviations from diagonal components of the Cauchy relations $g_{11}=c_{23}-c_{44}, g_{22}=c_{13}-c_{55}, g_{33}=c_{12}-c_{66}$ (Table ??) allow the interpretation of the shear stiffnesses and the transverse interaction coefficients as they are strongly related to the dominant type of bonding interactions. In crystals with strong ionic bonds, and particularly in those containing aspherical or highly polarizable constituents, the transverse interaction coefficients (for thenardite e.g. $c_{13}$ ) dominate considerably over the corresponding shear stiffness (for thenardite e.g. $c_{55}$ ), resulting in positive deviations from the Cauchy relation. In contrast, strong covalent bonds or other bonds with preferential orientations give rise to larger shear stiffnesses leading to small or negative $g_{i i}$. In thenardite, the $g_{i i}$ are quite similar and positive, which is consistent with a structure bulit up of constituents which are bound mainly by ionic interactions. This is similar to other sulphates, such as gypsum [? ?] and retgersite [? ] (Table ??).

A comparison of the elastic behaviour of different compounds can be based on the invariant and dimensionless quantity $\Delta=\left(g_{11}+g_{22}+g_{33}\right) / C$ where $C=$ $\left(c_{11}+c_{22}+c_{33}+c_{12}+c_{13}+c_{23}+c_{44}+c_{55}+c_{66}\right) / 9$ denotes the mean elastic stiffness. The mean elastic stiffnesses of thenardite. gypsum and retgersite are rather similar (Table ??) despite the presence of significant structural differences. The $\Delta$-values of thenardite, gypsum and retgersite are all positive, indicating again the mainly ionic bonding system of these crystal structures.

The observed directional anisotropy of the elastic stiffness of thenardite at ambient conditions is predicted to persist both at lower and at higher temperatures. 
The thermal expansion of thenardite and of the other two phases is strongly anisotropic. Thenardite expands more along the $c$ direction. This is consistent with $c_{33}$ being smaller than $c_{11}$ and $c_{22}$. Phase II exhibits anomalous thermal contraction along the $b$-axis. This effect is likely to be the cause of the predicted elastic instability of this phase above $475 \mathrm{~K}$. The phases II and III also differ from thenardite by their significantly higher entropies. This prediction is consistent with the calorimetric results of ? ]. The calorimetric $\mathrm{S}_{298}$ values for the phases $\mathrm{V}$ and III of 149.6 and $154.9 \mathrm{~J} / \mathrm{K} / \mathrm{mol}$, respectively, compare well with our results $(151.6$ and $157.5 \mathrm{~J} / \mathrm{K} / \mathrm{mol}$, respectively). The higher entropies of the phases II and III could be responsible for their metastable crystallisation as the decomposition products of the hexagonal phase [CHECK].

Turning to consider the results of the calculations, the derived force-field model was applied to the calculation of the structure of the three polymorphs as a function of temperature. The predicted lattice parameters of the phases II, III and V are compared to the available experimental data in Table ??. The relative errors between the predicted and the experimental lattice parameters are below $1 \%$ for phases III and V, with the exception of the $a$ parameter of thenardite, which has a deviation of $1.7 \%$. It appears that force-fields fail to capture the anisotropy of thenardite correctly as no fit could be found that reproduced all lattice parameters uniformly, regardless of which potential parameters are varied. The fact that quantum mechanical calculations yield a more uniform systematic error suggests that the fixed charge distribution that imposes equal charges on all oxygen atoms may be the limitation within the forcefield description.

The present model allows the simulation of the phases III and V up to temperatures of at least $600 \mathrm{~K}$. This exceeds the known stability range of the phases III and $\mathrm{V}$ and thus for these two phases we were able to directly compare the predicted lattice constants with those measured experimentally. However, this was not so for phase II. According to ? ] phase II appears as a metastable decomposition product of the hexagonal phase (phase I) at about $503 \mathrm{~K}$. On further cooling to $493 \mathrm{~K}$ phase II transforms into phase III. The structural parameters of this phase were measured at $493 \mathrm{~K}$. Our model predicted this phase to become dynamically unstable just above $485 \mathrm{~K}$. Thus we were not able to directly compare the predicted lattice parameters of phase II with those determined experimentally at the appropriate temperature. However, the error in the lattice parameters still remains below $1.5 \%$. The different behaviour exhibited by phase II may be linked to the observation that $b$ lattice parameter exhibits negative thermal expansion.

Finally, we can compare the relative thermodynamics of the different polymorphs of $\mathrm{Na}_{2} \mathrm{SO}_{4}$. Based on quantum mechanical calculations using the PBEsol functional the stable phase is correctly predicted to be phase $\mathrm{V}$, with phase III being $3.7 \mathrm{~kJ} / \mathrm{mol}$ higher based on the internal energy. This agrees well with experimental estimates of the enthalpy change for the $\mathrm{V}$ to III transition, which are close to $3 \mathrm{~kJ} / \mathrm{mol}[?]$. Based on the DFT calculations phase II is found to be slightly lower in energy than phase III by $1.2 \mathrm{~kJ} / \mathrm{mol}$, in contradiction to the apparent order of stability based on the temperatures at which the polymorphs appear. However, the experimental enthalpy difference remains unknown. Using the free energy derived force-field the enthalpy difference between phases $\mathrm{V}$ and III is $5.0 \mathrm{~kJ} / \mathrm{mol}$, but with the wrong sign (i.e. phase III is more stable than phase V). If the free energy difference is used instead of the enthalpy then the difference increases to 5.4 and $6.7 \mathrm{~kJ} / \mathrm{mol}$ at 0 $\mathrm{K}$ and $298 \mathrm{~K}$, respectively. This indicates that although the absolute relative thermodynamics of phases $\mathrm{V}$ and III are incorrect, the trend that III becomes increasingly stable relative to $\mathrm{V}$ with increasing temperature is reproduced.

\section{Conclusions}

The observed good agreement between the measured and theoretically calculated $c_{i j}$ and $p_{i j}$ confirms that DFT is able to predict the elastic stiffness behaviour of moderately soft minerals. In the present case the agreement between the results of the DFT model calculations and experiment is significantly better that obtained by us in our earlier study [?], where we have investigated the piezoelastic properties of $\mathrm{NiSO}_{4} \cdot 6 \mathrm{H}_{2} \mathrm{O}$, a more compressible compound, whose structure-property relations are significantly determined by hydrogen bonds.

The current study shows that fitting based on free energy minimisations using structural and elastic data 
as observables leads to force-field models which can predict piezoelastic coefficients for compressible compounds with a similar accuracy to DFT calculations. However, the prediction of an accurate phase diagram for this system is beyond the present force-field model, while quantum mechanical calculations exhibit greater promise, at least for the two lowest energy phases.

\section{Acknowledgments}

The authors thank H. Siegert and J. Schreuer for providing their computer programs for the evaluation of the piezoelastic constants and the RUS spectra, respectively. The financial support from the Helmholtz Society (VHVI 313), the Deutsche Forschungsgemeinschaft (Grants Wi-1232/27-1 and HA5137/1) and BMBF (Mineral Surfaces: From Atomic Processes to Geotechnology) is gratefully acknowledged. JDG thanks the Australian Research Council for funding. 
